\title{
Magnetohydrodynamic waves in the pulsar magnetosphere
}

\author{
V. Urpin ${ }^{1,2}$ \\ 1 INAF - Osservatorio Astrofisico di Catania, via S. Sofia 78, 95123 Catania, Italy \\ e-mail: vadim.urpin@uv.es \\ 2 A.F. Ioffe Institute of Physics and Technology and Isaac Newton Institute of Chile, Branch in St. Petersburg, \\ 194021 St. Petersburg, Russia
}

Received 8 September 2011 / Accepted 7 October 2011

\begin{abstract}
Context. MHD waves can be responsible for plasma fluctuations and short-term variations of the pulsar emission.

Aims. We consider the properties of plane and cylindrical MHD waves that can exist in the force-free magnetosphere. Methods. Waves are considered by means of a linear analysis of the force-free MHD equations.

Results. We argue that these particular types of MHD waves can exist in the magnetosphere of pulsars. These waves are closely related to the Alfvén waves of the standard magnetohydrodynamics modified by the force-free condition and non-zero charge density. We derive the dispersion equation fror magnetospheric waves and show that the wave periods are likely within the range $\sim 10^{-2}-10^{-6} \mathrm{~s}$ depending on the magnetospheric parameters.
\end{abstract}

Key words. stars: oscillations - stars: neutron - magnetohydrodynamics (MHD) - stars: magnetic field - pulsars: general

\section{Introduction}

The magnetospheres of pulsars consist of electron-positron plasma with, possibly, some amounts of ions. This plasma can affect the radiation produced in the inner region of the magnetosphere or at the stellar surface. Therefore, understanding the properties of a magnetosphere is of crucial importance for the interpretation of observations. The growing observational data on spectra and pulse profiles of isolated pulsars prompt continued improvement of theoretical theoretical models of the pulsar magnetosphere (see, e.g., Goodwin et al. 2004; Contopoulos et al. 1999; Komissarov 2006). Apart from a quasi-static structure, however, various non-stationary phenomena in the magnetosphere may affect the radiation. Electromagnetic waves in pulsar plasma have been studied extensively over the past few decades. The properties of the low-frequency electromagnetic waves are of central importance for understanding the underlying processes in the formation of the radio spectrum. Early studies mainly concentrated on the relativistic plasma flow, assuming a cold or only mildy relativistic distribution of electrons and positrons in the plasma rest frame (see, e.g., Melrose 1996, and reference therein). Low frequency waves were studied by Arons \& Barnard (1986), where many of the results of the previous studies were rederived and generalized. In all these cases, the plasma was assumed to be one-dimensional which is justified for plasma in a strong magnetic field. The electrostatic oscillations with a low frequency have been studied recently by Mofiz et al. (2011), who found that the thermal and magnetic pressures can generate oscillations that propagate in the azimuthal direction near the equator.

Magnetohydrodynamic waves in the pulsar magnetosphere are studied in less detail. Perhaps the only exception are diocotron modes, which are the non-neutral plasma analog of the Kelvin-Helmholtz modes. These modes have been studied extensively in the context of laboratory plasma devices (see, e.g., Levy 1965; Davidson 1990; Davidson \& Felice 1998). However, diocotron modes can occur also in charged pulsar magnetospheres. The existence around pulsars of a differentially rotating equatorial disc with non-vanishing charge density could trigger a shearing instability of diocotron modes (Petri et al. 2002). In the non-linear regime, the diocotron instability can cause diffusion of the charged particles across the magnetic field lines outwards (Petri et al. 2003). This turbulent charge transport could bear on the problem of electric current closure in the pulsar inner magnetosphere. The role of a diocotron instability in causing drifting sub-pulses in radio pulsar emission has been considered by Fung et al. (2006). Note that the diocotron modes should be substantially suppressed in a neighbourhood of the light cylinder where relativistic effects become important (Petri 2007). The non-axisymmetric diocotron instability has been observed in 3D numerical modelling of the pulsar magnetosphere by Spitkovski \& Arons (2002). The properties of MHD waves should be very particular in the magnetosphere pulsars since the electromagnetic energy density in the magnetosphere is greater than the kinetic and thermal energy density for typical values of the magnetic field. This suggests that over much of the magnetosphere, the force-free equation will be a good approximation for various MHD phenomena, including waves. In this Letter we consider MHD waves that can exist in the pulsar magnetosphere.

\section{Basic equations}

Despite uncertainties in the estimate of many parameters, plasma in the pulsar magnetosphere is likely collisional and the Coulomb mean free path of electrons (and positrons) is shorter than the characteristic length scale. Therefore, the magnetohydrodynamic description can be justified in this plasma. The partial MHD momentum equation for the electrons and positrons 
can be obtained in the standard way - multiplying the Boltzmann kinetic equation by the velocity and integrating over velocity (see, e.g., Braginskii 1965). The sum of electron and positron momentum equations yields the well-known force-free condition if one neglects terms proportional electron mass and gas pressure,

$\rho_{\mathrm{e}} \boldsymbol{E}+\frac{1}{c} \boldsymbol{j} \times \boldsymbol{B}=0$,

where $\rho_{\mathrm{e}}=e\left(n_{\mathrm{p}}-n_{\mathrm{e}}\right)$ is the charge density. Taking the difference between electron and positron momentum equations, one obtains Ohm's law in the magnetosphere

$\boldsymbol{j}=\rho_{\mathrm{e}} \boldsymbol{V}+\sigma\left(\boldsymbol{E}+\frac{\boldsymbol{V}}{c} \times \boldsymbol{B}\right)$,

where $\sigma=e^{2} n_{\mathrm{p}} \tau_{\mathrm{e}} / m_{\mathrm{e}}$ is the conductivity and $\boldsymbol{V}$ is the plasma velocity.

Equations (1) and (2) can be rewritten as

$\rho_{\mathrm{e}} \boldsymbol{E}^{*}+\frac{1}{c} \boldsymbol{J} \times \boldsymbol{B}=0, \quad \boldsymbol{J}-\sigma \boldsymbol{E}^{*}=0$,

where

$\boldsymbol{E}^{*}=\boldsymbol{E}+\frac{\boldsymbol{V}}{c} \times \boldsymbol{B}, \quad \boldsymbol{J}=\boldsymbol{j}-\rho_{\mathrm{e}} \boldsymbol{V}$.

If $\rho_{\mathrm{e}} \neq 0$, one can exclude $\boldsymbol{E}^{*}$ from Eq. (3) and obtain the equation containing $\boldsymbol{J}$ alone,

$\boldsymbol{J}+\frac{\sigma}{c \rho_{\mathrm{e}}} \boldsymbol{J} \times \boldsymbol{B}=0$

It follows immediately from this equation that $\boldsymbol{J}=0$. Then, Eq. (3) yields $\boldsymbol{E}^{*}=0$. Hence, we have

$\boldsymbol{j}=\rho_{\mathrm{e}} \boldsymbol{V}, \quad \boldsymbol{E}=-\frac{\boldsymbol{V}}{c} \times \boldsymbol{B}$.

These equations imply that the force-free condition (1) and Ohm's law (2) are equivalent to the conditions that the magnetic field is frozen-in and the only advective current exists in the magnetosphere. Or, in other words the force-free condition (1) and Ohm's law (2) are compatible in a charged plasma only if the electric current is advective and the magnetic field is frozen-in. Note that expression (6) can be applied only in the regions where $\rho_{\mathrm{e}} \neq 0$.

Equation (6) should be complemented by the partial continuity equations for electrons and positrons

$\frac{\partial n_{\alpha}}{\partial t}+\nabla \cdot\left(n_{\alpha} \boldsymbol{V}_{\alpha}\right)=\Gamma_{\alpha}=\Gamma_{\alpha}^{(+)}-\Gamma_{\alpha}^{(-)}$,

where $\Gamma_{\alpha}^{(+)}$and $\Gamma_{\alpha}^{(-)}$are the rates of generation and annihilation of particles of the sort $\alpha$ and $\boldsymbol{V}_{\alpha}$ is their partial velocity. Since electrons and positrons are generated and annihilate in pairs, we have $\Gamma_{\mathrm{e}}^{(+)}=\Gamma_{\mathrm{p}}^{(+)}$and $\Gamma_{\mathrm{e}}^{(-)}=\Gamma_{\mathrm{p}}^{(-)}$. The difference of the positron and electron equations yield the charge conservation law,

$\frac{\partial \rho_{\mathrm{e}}}{\partial t}+\nabla \cdot \boldsymbol{j}=0$

The sum of partial Eq. (7) yields the equation for the total number density

$\frac{\partial n}{\partial t}+\nabla \cdot(n \boldsymbol{V})=2 \Gamma_{\mathrm{e}}$

\section{Equation for MHD waves in the pulsar magnetosphere}

MHD processes in the force-free pulsar magnetosphere are governed by Eqs. (6), (8), and (9) complemented by the Maxwell equations

$$
\begin{aligned}
\nabla \cdot \boldsymbol{E} & =4 \pi \rho_{\mathrm{e}}, \quad \nabla \times \boldsymbol{E}=-\frac{1}{c} \frac{\partial \boldsymbol{B}}{\partial t}, \\
\nabla \cdot \boldsymbol{B} & =0, \quad \nabla \times \boldsymbol{B}=\frac{1}{c} \frac{\partial \boldsymbol{E}}{\partial t}+\frac{4 \pi}{c} \boldsymbol{j}, \\
\boldsymbol{j} & =\rho_{\mathrm{e}} \boldsymbol{V}, \quad \boldsymbol{E}=-\frac{\boldsymbol{V}}{c} \times \boldsymbol{B}, \\
\frac{\partial n}{\partial t} & +\nabla \cdot(n \boldsymbol{V})=2 \Gamma_{\mathrm{e}}, \quad \frac{\partial \rho_{\mathrm{e}}}{\partial t}+\nabla \cdot \boldsymbol{j}=0 .
\end{aligned}
$$

The force-free MHD phenomena are very particular and this point can be illustrated by considering linear MHD waves. We assume that the electric and magnetic fields are equal to $\boldsymbol{E}_{0}$ and $\boldsymbol{B}_{0}$ in the unperturbed magnetosphere. The corresponding electric current and charge density are $\boldsymbol{j}_{0}$ and $\rho_{\mathrm{e} 0}$, respectively. For the sake of simplicity, we neglect hydrodynamic motions in the magnetosphere and focus on the effects caused by electric currents. Linearizing Eq. (10), we obtain the set of linear equations for waves of a small amplitude. Small perturbations will be indicated by subscript 1 . We here consider the waves with a short wavelength and space-time dependence $\alpha \exp (\mathrm{i} \omega t-\mathrm{i} \boldsymbol{k} \cdot \boldsymbol{r})$, where $\omega$ and $\boldsymbol{k}$ are the frequency and wave vector, respectively. These waves exist if their wavelength $\lambda=2 \pi / k$ is short compared to the characteristic length scale of the magnetosphere, $L$. For these perturbations Eq. (10) takes the form

$$
\begin{aligned}
\boldsymbol{k} \cdot \boldsymbol{E}_{1} & =4 \pi \rho_{\mathrm{e} 1}, \quad c \boldsymbol{k} \times \boldsymbol{E}_{1}=\omega \boldsymbol{B}_{1}, \quad \boldsymbol{k} \cdot \boldsymbol{B}_{1}=0 \\
c \boldsymbol{k} \times \boldsymbol{B}_{1} & =4 \mathrm{i} \pi \boldsymbol{j}_{1}-\omega \boldsymbol{E}_{1}, \quad \boldsymbol{j}_{1}=\rho_{\mathrm{e} 0} \boldsymbol{V}_{1} \\
c \boldsymbol{E}_{1} & =-\boldsymbol{V}_{1} \times \boldsymbol{B}_{0}, \quad \omega \rho_{\mathrm{e} 1}=\boldsymbol{k} \cdot \boldsymbol{j}_{1} \\
\mathrm{i} \omega n_{1} & -\mathrm{i} \boldsymbol{k} \cdot\left(n_{0} \boldsymbol{V}_{1}\right)=2 \Gamma_{\mathrm{e} 1} .
\end{aligned}
$$

We study only MHD modes with $\omega \ll c k$ because one can split electromagnetic and hydrodynamic modes in this case. At $\omega \sim c k$, a consideration becomes quite cumbersome because electromagnetic and hydromagnetic modes are strongly coupled. We focus on understanding the nature and qualitative features of magnetospheric waves, rather than the direct relevance for observational implications, and a consideration of the simple particular case $\omega \ll c k$ can help in that. For the sake of simplicity, we consider waves in the region where $\Gamma_{\mathrm{e}} \approx 0$. Substituting the frozen-in condition $\boldsymbol{E}=-\boldsymbol{V} \times \boldsymbol{B} / c$ into the equation $c \nabla \times \boldsymbol{E}=-\partial \boldsymbol{B} / \partial t$ and linearizing the obtained induction equation, we have

$\mathrm{i} \omega \boldsymbol{B}_{1}=\mathrm{i} \boldsymbol{B}_{0}\left(\boldsymbol{k} \cdot \boldsymbol{V}_{1}\right)-\mathrm{i} \boldsymbol{V}_{1}\left(\boldsymbol{k} \cdot \boldsymbol{B}_{0}\right)-\left(\boldsymbol{V}_{1} \cdot \nabla\right) \boldsymbol{B}_{0}$.

Eliminating $\boldsymbol{j}_{1}$ from two equations in the second line of Eq. (11), we obtain the following expression for $\boldsymbol{V}_{1}$

$\boldsymbol{V}_{1}=-\frac{\mathrm{i}}{4 \pi \rho_{\mathrm{e} 0}}\left(c \boldsymbol{k} \times \boldsymbol{B}_{1}+\omega \boldsymbol{E}_{1}\right)$

Substituting the expression for $\boldsymbol{E}_{1}$ obtained from the linearized frozen-in condition and neglecting terms terms of the order of $(\omega / c k)^{2}$, we have

$\boldsymbol{V}_{1}+\frac{\mathrm{i} \omega}{4 \pi c \rho_{\mathrm{e} 0}} \boldsymbol{B}_{0} \times \boldsymbol{V}_{1}=-\frac{\mathrm{i} c}{4 \pi \rho_{\mathrm{e} 0}} \boldsymbol{k} \times \boldsymbol{B}_{1}$. 
The perturbation of the charge density can be calculated from the equation $\rho_{\mathrm{e} 1}=\nabla \cdot \boldsymbol{E}_{1} / 4 \pi$, then

$\rho_{\mathrm{e} 1}=\frac{1}{4 \pi c}\left[\mathrm{i} \boldsymbol{B}_{0} \cdot\left(\boldsymbol{k} \times \boldsymbol{V}_{1}\right)+\boldsymbol{V}_{1} \cdot\left(\nabla \times \boldsymbol{B}_{0}\right)\right]$.

Eliminating $\boldsymbol{B}_{1}$ from Eqs. (12) and (14) in favour of $\boldsymbol{V}_{1}$ and neglecting again terms $\sim(\omega / c k)^{2}$, we obtain the following equation for $V_{1}$

$4 \pi \rho_{\mathrm{e} 0} \boldsymbol{V}_{1}=\frac{c}{\omega} \boldsymbol{k} \times\left[\left(\boldsymbol{V}_{1} \cdot \nabla\right) \boldsymbol{B}_{0}-\mathrm{i} \boldsymbol{B}_{0}\left(\boldsymbol{k} \cdot \boldsymbol{V}_{1}\right)+\mathrm{i} \boldsymbol{V}_{1}\left(\boldsymbol{k} \cdot \boldsymbol{B}_{0}\right)\right.$.

The scalar production of this equation and vector $\boldsymbol{k}$ yields the condition

$\left(\boldsymbol{k} \cdot \boldsymbol{V}_{1}\right)=0$.

This equation implies that the longitudinal waves (with $\boldsymbol{k} \cdot \boldsymbol{V}_{1} \neq$ 0 ) cannot exist in the force-free magnetosphere. Only transverse waves with the velocity perpendicular to the wave vector $\left(\boldsymbol{k} \cdot \boldsymbol{V}_{1}=0\right)$ can propagate in this magnetosphere. We have for transverse waves from Eq. (16)

$4 \pi \rho_{\mathrm{e} 0} \boldsymbol{V}_{1}-\mathrm{i} \frac{c}{\omega}\left(\boldsymbol{k} \cdot \boldsymbol{B}_{0}\right) \boldsymbol{k} \times \boldsymbol{V}_{1}=\frac{c}{\omega} \boldsymbol{k} \times\left(\boldsymbol{V}_{1} \cdot \nabla\right) \boldsymbol{B}_{0}$.

Equation (18) is the basic equation governing linear MHD waves in the force-free magnetosphere. Note that transformations from Eqs. (11) to (18) were made taking into account terms of the two lowest orders in $\lambda / L$. The term on the r.h.s. of Eq. (18) is, in general, of the order of $\lambda / L$ compared to the second term on the l.h.s. However, this is not the case if $\boldsymbol{k}$ is approximately perpendicular to $\boldsymbol{B}_{0}$ when the term on the r.h.s. becomes dominating.

\section{MHD waves in the pulsar magnetosphere}

Plane waves. Consider initally the case of plane waves with $(\boldsymbol{k} \cdot \boldsymbol{B}) \gg \lambda / L$ where we can meglect the term on the r.h.s. of Eq. (18). We have for these waves

$4 \pi \rho_{\mathrm{e} 0} \boldsymbol{V}_{1}-\mathrm{i} \frac{c}{\omega}\left(\boldsymbol{k} \cdot \boldsymbol{B}_{0}\right) \boldsymbol{k} \times \boldsymbol{V}_{1}=0$.

The dispersion relation corresponding to this equation is

$\omega^{2}=\frac{c^{2} k^{2}(\boldsymbol{k} \cdot \boldsymbol{b})^{2}}{\Omega_{\mathrm{m}}^{2}}$,

where $\Omega_{\mathrm{m}} 4 \pi c \rho_{\mathrm{e} 0} / B_{0}$ and $\boldsymbol{b}=\boldsymbol{B}_{0} / B_{0}$. It is convenient to express the characteristic frequency $\Omega_{\mathrm{m}}$ in terms of the Goldreich-Julian charge density, $\rho_{\mathrm{GJ}}=\Omega B_{0} / 2 \pi c$ where $\Omega$ is the angular velocity of a neutron star. Then, we have $\Omega_{\mathrm{m}}=\xi \Omega$ and $\xi=\rho_{\mathrm{e} 0} / \rho_{\mathrm{GJ}}$, and the dispersion Eq. (20) can be rewritten as

$\omega= \pm c(\boldsymbol{k} \cdot \boldsymbol{b}) \frac{c k}{\xi \Omega}$.

This equation describes the new mode of oscillations that can exist in the force-free pulsar magnetosphere. Equation (21) is like the dispersion equation for whistlers in "standard" plasma. However, there is a principle difference between the considered waves and whistlers since Eq. (21) contains the charge density $\rho_{\mathrm{e} 0}=e\left(n_{\mathrm{p} 0}-n_{\mathrm{e} 0}\right)$, whereas the dispersion relation for whistlers is determined by $e n_{\mathrm{e} 0}$. Therefore, the magnetospheric waves do not exist if $\rho_{\mathrm{e} 0}=0$, but whistlers can propagate in neutral plasma. The frequency of magnetospheric waves is higher because it is generally believed that $\left|n_{\mathrm{p}}-n_{\mathrm{e}}\right| \ll n_{\mathrm{e}}$ in the magnetosphere.
Deriving Eq. (21), we assumed that $\omega \ll c k$. Therefore, the considered modes exist if

$\xi \Omega>c(\boldsymbol{k} \cdot \boldsymbol{b})$.

This condition can be satisfied for plane waves with the wave vector almost (but not exactly) perpendicular to the magnetic field, for which the scalar production $(\boldsymbol{k} \cdot \boldsymbol{b})$ is small. For example, if the angle between $\boldsymbol{k}$ and $\boldsymbol{B}_{0}$ is $(\pi / 2-\delta), \delta \ll \pi / 2$, then Eq. (22) is satisfied if

$\delta<\xi \Omega / c k$.

Note that generally, magnetospheric waves can exist even if $\delta \sim 1$ but our consideration does not apply to this case.

Cylindrical waves. Let us assume that the basic magnetic configuration of a neutron star is dipole and consider a particular sort of waves that can exist in a neighbourhood of the magnetic axis. In this neighbourhood, the field is approximately parallel (or antiparallel) to the axis but the field component perpendicular to the axis is small. We will mimic the magnetic geometry near the axis by a cylindrical configuration with the magnetic field in the $z$-direction. Introducing the cylindrical coordinates $(s, \varphi, z)$ with the unit vectors $\left(\boldsymbol{e}_{s}, \boldsymbol{e}_{\varphi}, \boldsymbol{e}_{z}\right)$, we can model the magnetic field as $\boldsymbol{B}_{0}=B_{0}(s) \boldsymbol{e}_{z}$. Consider the special case of perturbations with the wavevector perpendicular to the magnetic field, $\boldsymbol{k}=\left(k_{s}, k_{\varphi}, 0\right)$ where $k_{\varphi}=m / s$ and $m$ is integer. Note that even though we used a short wavelength approximation deriving Eq. (18), $m$ should not be large for cylindrical waves because the cylindrical symmetry of the basic state is assumed. For these perturbations, Eq. (18) reads

$4 \pi \rho_{\mathrm{e} 0} \boldsymbol{V}_{1}=\frac{c}{\omega} \boldsymbol{k} \times \boldsymbol{e}_{z} V_{1 s} \frac{\mathrm{d} B_{0}}{\mathrm{~d} s}$.

Taking the radial component of this equation, we obtain the dispersion relation for cylindrical waves in the form

$\omega=\frac{c k_{\varphi}}{4 \pi \rho_{\mathrm{e} 0}} \frac{\mathrm{d} B_{0}}{\mathrm{~d} s}$.

Cylindrical waves may exist only if $m \neq 0$. These waves propagate around the magnetic axis with the velocity $\left(c / 4 \pi \rho_{\mathrm{e} 0}\right)\left(\mathrm{d} B_{0} / \mathrm{d} s\right)$ and period

$P_{m}=\frac{2 \pi}{\omega}=\frac{8 \pi^{2} s \rho_{\mathrm{e} 0}}{m c\left(\mathrm{~d} B_{0} / \mathrm{d} s\right)}$.

If we represent the unperturbed charge density as $\xi \rho_{\mathrm{e} 0}$, then

$P_{m}=\frac{2 \xi}{\alpha m} P\left(\frac{s \Omega}{c}\right)^{2}$,

where $P=2 \pi / \Omega$ is the rotation period of a pulsar and $\alpha=$ $\mathrm{d} \ln B_{0} / \mathrm{d} \ln s$. The parameter $\alpha$ depends on the magnetic configuration. We can estimate it assuming that the poloidal field is approximately dipole near the axis. The radial and polar components of the dipole field in the spherical coordinates $(r, \theta, \varphi)$ are

$B_{r}=B_{\mathrm{p}}\left(\frac{a}{r}\right)^{3} \cos \theta, \quad B_{\theta}=\frac{1}{2} B_{\mathrm{p}}\left(\frac{a}{r}\right)^{3} \sin \theta$,

where $B_{\mathrm{p}}$ is the polar strength of the magnetic field at the neutron star surface and $a$ is the stellar radius (see, e.g., Urpin et al. 1994). The radial field component is much greaterthan the polar one near the magnetic axis and, hence, $\alpha \approx \mathrm{d} \ln B_{r} / \mathrm{d} \ln s$. Taking into account that $r^{2}=s^{2}+z^{2}$ and $s \ll z$ in the neighbourhood 
of the axis, we obtain with accuracy in terms of the lowest order in $s / r$ that $\alpha=-4 s^{2} / r^{2}$. Then, substituting this estimate into Eq. (27), we have

$P_{m}=\frac{\xi}{2 m} P\left(\frac{r \Omega}{c}\right)^{2}$.

It turns out that the period of cylindrical waves does not depend on the distance from the magnetic axis if $s \ll r$ but depends on the height above the magnetic pole. Therefore, at any given height, perturbations rotate rigidly around the axis with the period $P_{m}$. For example, $P_{m}$ near the polar spot at the surface is approximately

$P_{m} \approx 6.3 \times 10^{-6} \frac{\xi}{m} P_{0.01}^{-1}$,

where $P_{0.01}=P / 0.01 \mathrm{~s}$. The period is longer at a higher height, $r>a$.

We have considered the cylindrical waves only for the dipole geometry. Note that in real pulsars, the magnetic field can depart from a simple dipole geometry and may have a very complex topology even with small-scale components (see, e.g., Bonanno et al. 2003, 2005, 2006). The mechanism of formation of these complex magnetic structures is related to the earliest stage of the neutron star life when the star is hydrodynamically unstable. Dynamo action induced by instabilities generates the magnetic field of various length scales that range from the stellar radius to a very short dissipative scale. These magnetic fields can be frozen into the crust that forms when the neutron star cools down. Owing to a high conductivity of the crust, the magnetic structures can survive a very long time. For example, structures with the length scale $\geq 1 \mathrm{~km}$ (magnetic spots on the surface) survive during the entire active life-time of pulsars (Urpin \& Gil 2004). The complex magnetic topology is inevitable in neutron stars and can be very important for the magnetospheric structure, particularly, in regions close to the star. It is likely, therefore, that cylindrical magnetic geometries similar to those considered in this section can exist in different regions of the magnetosphere.

\section{Discussion}

Our consideration shows that the particular type of waves can exist in the force-free pulsar magnetosphere. These waves are closely related to the Alfvén waves of the standard magnetohydrodynamics but they are substantially modified by the forcefree condition and non-vanishing electric charge density in the pulsar magnetosphere. Like Alfvén waves, the magnetospheric ones are transverse because plasma moves only in the direction perpendicular to the wavevector, $\boldsymbol{k} \cdot \boldsymbol{V}_{1}=0$. Our simplified analysis applies only to waves with $\boldsymbol{k}$ almost perpendicular to the magnetic field, $\boldsymbol{k} \cdot \boldsymbol{B} \ll k B$ but, in general, these waves can exist for any $\boldsymbol{k}$.

The frequency of magnetospheric waves depends on the ratio of the true and Goldreich-Julian charge densities, $\xi$. The pair plasma in the pulsar magnetosphere is likely created in a twostage process: primary particles are accelerated by an electric field parallel to the magnetic field near the poles up to extremely high energy and these produce a secondary, denser pair plasma via a cascade process (see, e.g., Michel 1982). The number density of this secondary plasma can exceed the Goldreich-Julian number density, $n_{\mathrm{GJ}}$, required to maintain a corrotation electric field and, hence, the factor $\xi$ can be large. Unfortunately, this factor is model-dependent and quite unsertain (see, e.g., Gedalin et al. 1998; Usov \& Melrose 1996) and can be different in different regions of the magnetosphere. Therefore, it is difficult to estimate the periods of waves with a high accuracy but, likely, they are within a range $10^{-6}-10^{-2} \mathrm{~s}$.

In our analysis, we neglected the destabilizing effects of electric currents and shear (that can exist, for example, because of differential rotation). These effects may lead to instability of the magnetospheric waves and exponential growth of small perturbations. Perhaps the instability caused by electric currents is more efficient in a very strong magnetic field. This sort of instability has received considerable attention in the literature on thermonuclear fusion. The principal features of the pulsar magnetosphere are the force-free condition and non-zero charge density. Nevertheless, in general, the current-driven instability can generate the magnetospheric waves. Another mechanism for generating these waves can be relevant for shear and/or differential rotation. It is well known that Alfvén waves can be generated in differentially rotating plasma with the magnetic field by the magnetorotational instability (Velikhov 1959). This mechanism can operate even if the magnetic field is strong and the plasma is magnetized (Urpin \& Rüdiger 2005). Likely, this type of mechanism also operate in those regions of the pulsar magnetosphere where plasma rotates differentially. The generation mechanisms of the magnetospheric waves will be considered elsewhere. Generation of waves will probably lead to fluctuations of the magnetospheric parameters and, hence, the emission of pulsars. Measurements of the time scale of these fluctuations can provide information regarding the physical conditions in the magnetosphere.

\section{References}

Arons, J., \& Barnard, J. 1986, ApJ, 302, 120

Bonanno, A., Rezzolla, L., \& Urpin, V. 2003, A\&A, 410, L33

Bonanno, A., Urpin, V., \& Belvedere, G. 2005, A\&A, 440, 199

Bonanno, A., Urpin, V., \& Belvedere, G. 2006, A\&A, 451, 1049

Braginskii, S. 1965, Rev. Plasma Phys., 1, 205

Contopoulos, I., Kazanas, D., \& Fendt, C. 1999, ApJ, 511, 351

Davidson, R. 1990, Physics of non-neutral plasmas (Addison-Wesley Publishing Company)

Davidson, R., \& Felice, G. 1998, Phys. Plasma, 5, 3497

Fung, P. K., Khechinashvili, D., \& Kuijpers, J. 2006, A\&A, 445, 779

Gedalin, M., Melrose, D., \& Gruman, E. 1998, PRE, 57, 3399

Goodwin, S., Mestel, J., Mestel, L., \& Wright, G. 2004, MNRAS, 349, 213

Komissarov, S. 2006, MNRAS, 367, 19

Levy, R. 1965, Phys. Plasma, 8, 1288

Melrose, D. 1996, Plasma Phys. Controlled Fusion, 39, 93

Michel, F. 1982, Rev. Mod. Phys., 54, 1

Mofiz, U., Amin, M., \& Shukla, P. 2011, Ap\&SS, in press [arXiv: 1108.4176]

Petri, J. 2007, A\&A, 469, 843

Petri, J., Heyvaerts, J., \& Bonazzola, S. 2002, A\&A, 287, 520

Petri, J., Heyvaerts, J., \& Bonazzola, S. 2003, A\&A, 411, 203

Spitkovski, A., \& Arons, J. 2002, ASP Conf. Ser., 271, 81

Urpin, V., \& Gil, J. 2004, A\&A, 415, 305

Urpin, V., \& Rüdiger, G. 2005, A\&A, 437, 23

Urpin, V., Chanmugam, G., \& Sang, Y. 1994, ApJ, 433, 780

Usov, V., \& Melrose, D. 1996, ApJ, 464, 306

Velikhov, E. 1959, Sov. Phys. JETP, 9, 995 